\title{
Effect of Al Addition on the Electronic structure of Hafnia by spatially resolved electron energy loss spectroscopy
}

\author{
Quan Li*, Jiyan Dai**, and Xingao Gong*** \\ *Department of Physics, The Chinese University of Hong Kong, Shatin, New Territory, Hong \\ Kong \\ ** Department of Applied Physics, The Hong Kong Polytechnic University, HomHum, Hong \\ Kong \\ *** Department of Physics, Fudan University, ShangHai, China
}

The scaling requirements predict the end of $\mathrm{SiO}_{2}$ as gate dielectric material in the complementary metal-oxide-semiconductor (CMOS) integrated circuit technology, and leads to extensive studies on the high-dielectric constant (high- $\kappa$ ) materials as potential replacements for $\mathrm{SiO}_{2}[1]$. Among these high- $\kappa$ dielectrics, pseudo-binary Hf-based materials appear as one of the most promising material system, which have demonstrated satisfactory electrical behavior. Compared to pure $\mathrm{HfO}_{2}$, the addition of a third element (usually $\mathrm{Si}$ or $\mathrm{Al}$ ) is expected to increase the $\mathrm{Si} /$ dielectric interfacial stability, the crystalline temperature of the material, the material's band gap and both the valance and conduction band offset to Si [2-3]. Although numerous papers have appeared in the past couple of years addressing the materials' physical/electrical properties, many of the results are controversial. Most importantly, one piece of fundamental information is incomplete - the local electronic structure(s) of material in the gate dielectric stack. Such information, including the material band gap, defect or defect-related states in the band gap, and the density of states in the valance and conduction band, is one of the most critical issues in understanding and further improving the electrical behavior of the gate dielectrics.

In the present work [4], we have carried out a systematic study on the electronic structure evolution of a series of hafnium aluminate thin films as a function of the Al concentration. The electron energy loss spectroscopy (EELS) in a scanning transmission electron microscope (STEM) provides a unique means to obtain such information, owing to the very localized electron probe, which is desired due to the finite thickness of the film and the existence of interface(s) in the gate dielectric stack. The hafnium aluminate films were deposited by pulse laser deposition using targets of the same material with different $\mathrm{Hf} / \mathrm{Al}$ ratio. HF-etched Si (100) wafers were used as the substrates. No interfacial layer is observed in-between the film and the substrate for the asdeposited samples (Figure 1). Together with the x-ray photoelectron spectroscopy, the valence electron energy loss spectrum (VEELS) of pure hafnia (Figure 2) discloses the existence of two occupied density of states in the material's band gap, which were suggested as related to charged oxygen vacancies and oxygen interstitials by first principle calculation. The density of states related to such defects significantly increase when the films are deposited at higher substrate temperatures. It is interesting to note that although $\mathrm{Al}$ addition has little effect on the band gap of pure $\mathrm{HfO}_{2}(5.7 \mathrm{eV}$ as measured by the VEELS), it can effectively passivate mid-gap levels induced by the oxygen vacancy and improve its electrical properties. Two other distinct changes are observed on the loss spectrum of pure $\mathrm{HfO}_{2}$ with the $\mathrm{Al}$ addition. One is the decrease in the oscillation strength of the $\sim 15 \mathrm{eV}$ excitation, and the other is the peak shifting and narrowing of the $\sim 26 \mathrm{eV}$ excitation when the Al concentration is increased and the film changes from pure hafnia to pure alumina. The origin of these changes will be discussed. 
References

[1] G. Wilk, R. Wallace, J. Anthony, J. Appl. Phys. 89, 5243 (2001)

[2] V. V. Afanas'ev, A. Stesmans, W. Tsai, Appl. Phys. Lett., 82245 (2003)

[3] J. Robertson, Eur. Phys. J. Appl. Phys. 28, 265 (2004)

[4] This work was supported by a grant from CERG under project No. CUHK402105. The authors thank Prof. R. F. Egerton and Prof. C. Colliex for stimulating discussions.

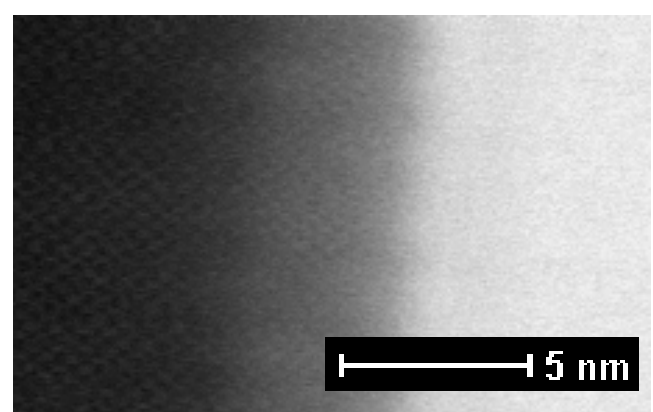

Figure 1. High resolution image take by high angle annular dark field detector showing the interface in-between the hafnium aluminate film and the Si substrate.

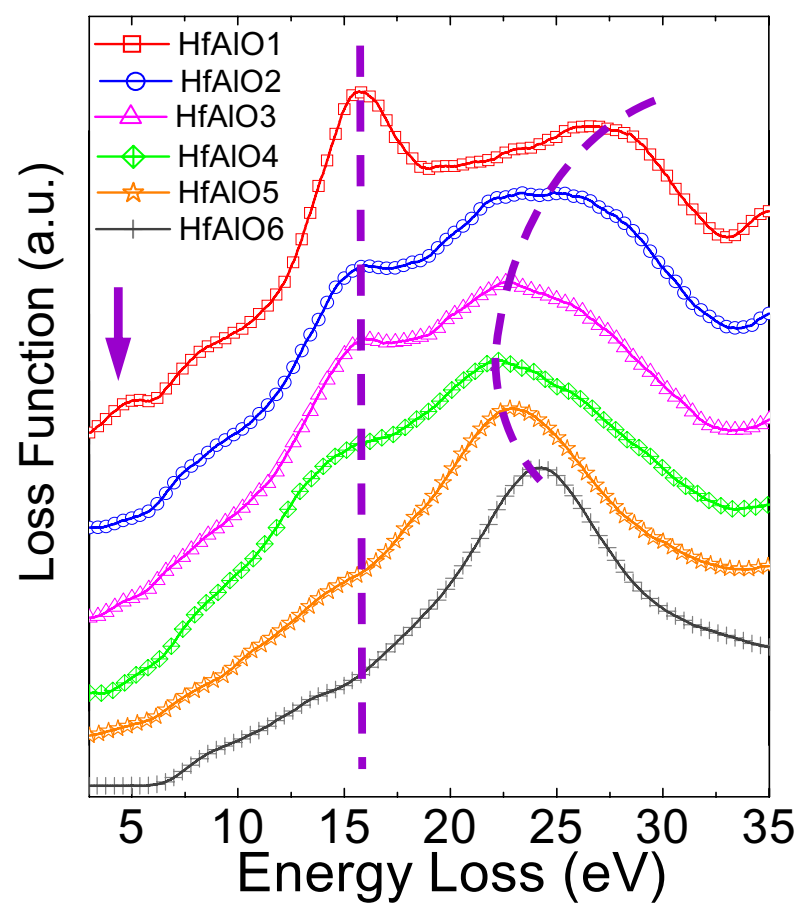

Figure 2. Loss functions of the hafnium aluminate films with Al concentration increase (from HfAlO1 to HfAlO6). The raw spectra were taken using the Gatan Imaging filtering (GIF) system attached to a Tecnai microscope (Tecnai 20ST). The spectra were taken at zero momentum transfer with energy resolution of $0.7 \mathrm{eV}$. 